\title{
Stem cell-based cartilage regeneration technology
}

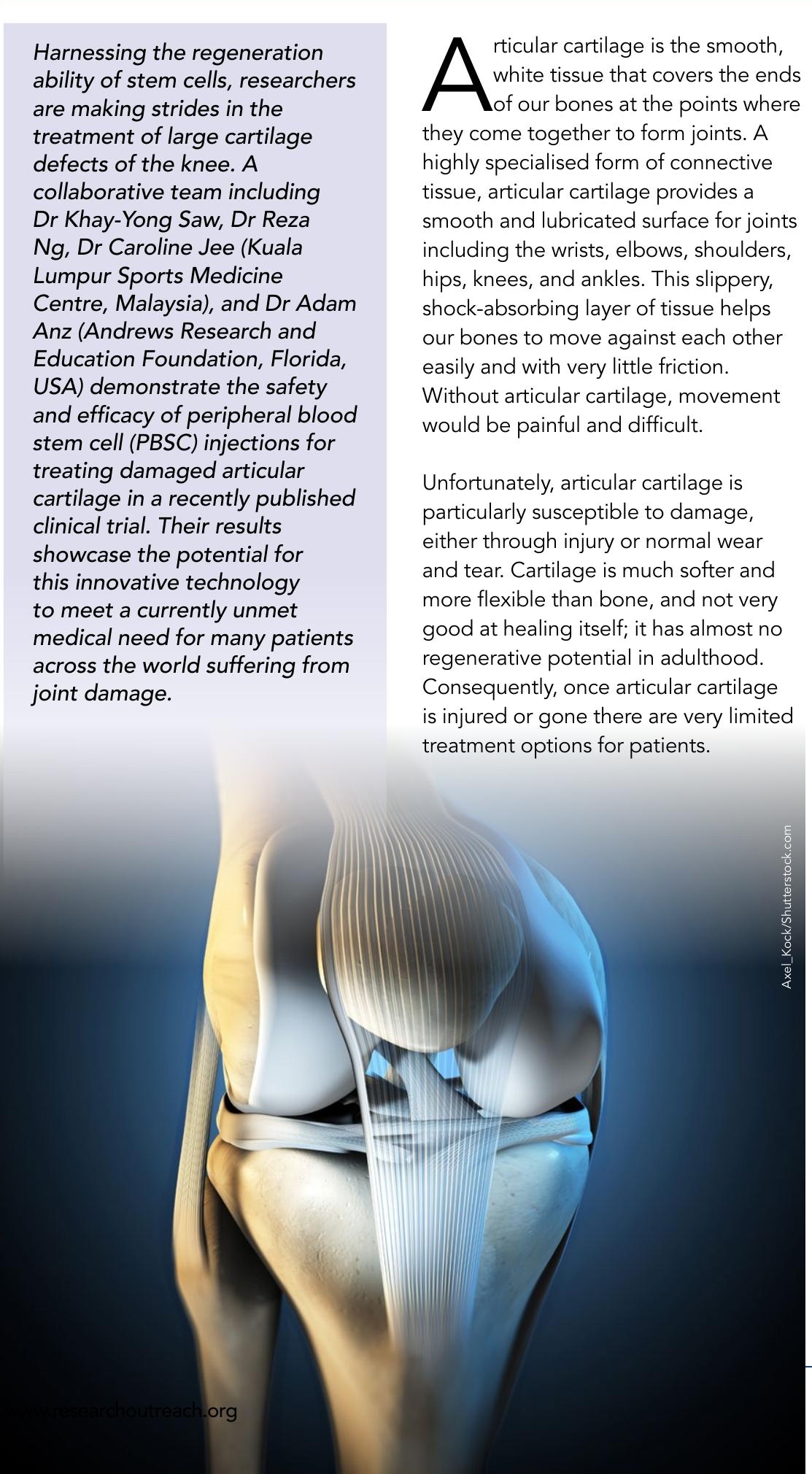

This is where innovative research from Dr Khay-Yong Saw, Dr Reza Ng, and Dr Caroline Jee (Kuala Lumpur Sports (Andrews Institute, Florida) comesin. Years of research, including a recently published clinical trial, shows that peripheral blood stem cell injections offer exciting potential as an effective way of treating major articular

cartilage damage.

CARTILAGE DEFECTS Articular cartilage damage can lead to chronic and painful symptoms including stiffness and swelling. Moving those joints becomes very painful and this damage can lead to arthritis later in life. There are limited surgical options available for people with damaged articular cartilage. The surgical goal is to stimulate the growth of new hyaline cartilage, the One approach, known as microfracture, attempts to encourage the growth of new articular cartilage via the creation of a new blood supply. This involves using a sharp tool to make holes in the oint surface, which brings a new blood supply, new cells, and stimulates a healing response. The same outcome is sometimes achieved through drilling or abrasion arthroplasty. Another method known as autologous chondrocyte implantation involves new cartilage cells being grown in a laboratory and then implanted back onto the joint. Some patients will also have cartilage transferred from a healthy non-weightbearing part of the bone area of the joint.

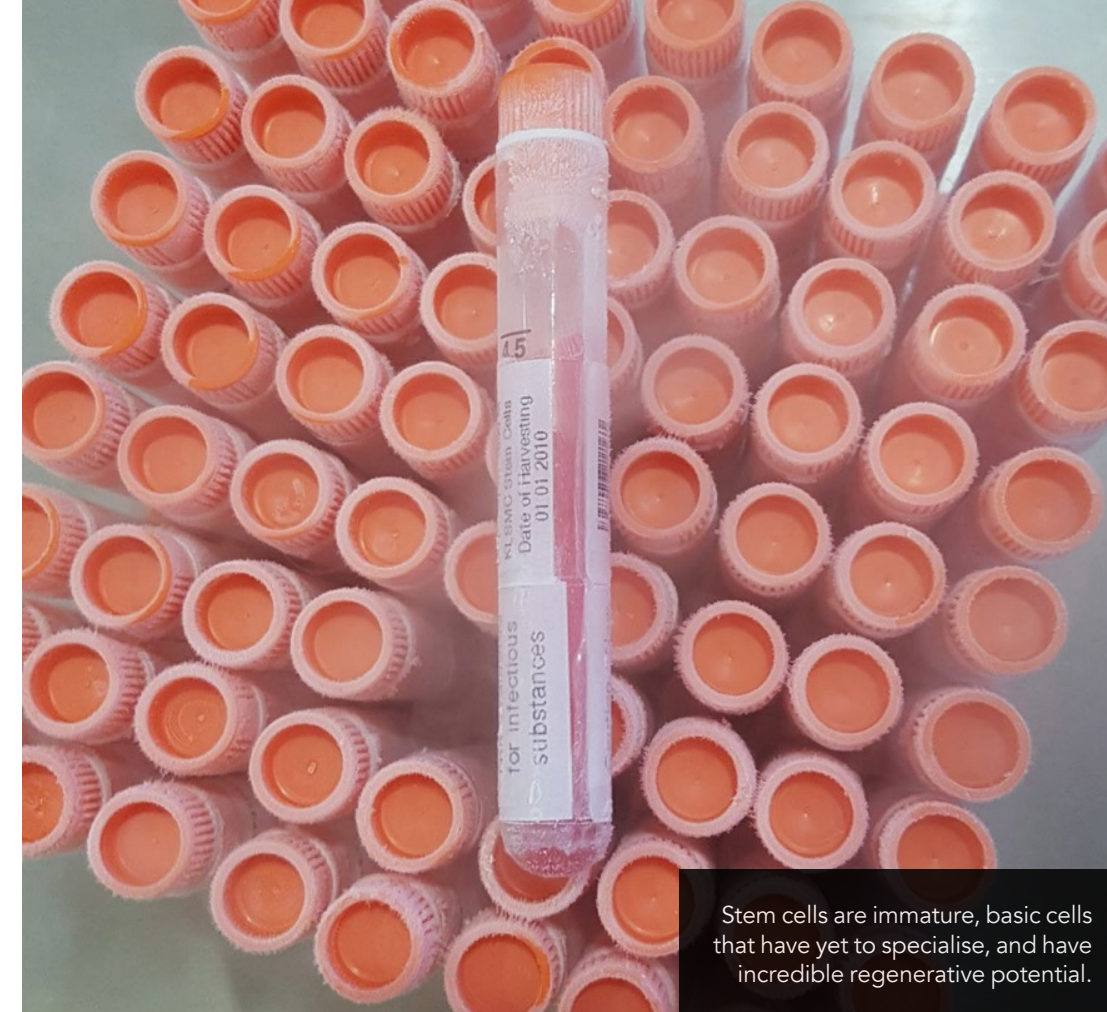

These methods all have their limitations however, and fully repairing articular medical need. Conseguiently many patients experience long and panful periods before undergoing a totat joint replacement.

\section{THE POWER OF STEM CELLS} Building on many years of research, a pioneering approach involving stem cell technology has been developed by Saw, Ng, Jee, and Anz. Their goal? To place stem cells at the damaged area of the joint to stimulate cartilage growth
(called chondrogenesis).

This innovative technology has been developed to enable high-quality repair and regeneration of articular cartilage. Articular Regeneration Tech (KLSMC aim is to achieve chondrogenesis by exploiting the natural healing qualities of a patient's own peripheral blood stem cells (PBSC). Formed in the bon marrow, PBSC are the precursor of all the different types of blood cell. Early work carried out by Saw and colleag Following their successful findings in carried out a series of studies inues humans to find out if their damaged knee joints could be restored using their KART technology. The researchers accessed the damaged knees using arthroscopic subchondral drilling (into the layer of bone just beneath the cartilage), a long-standing method of stimulating the bone marrow. Subsequent (postoperative) intraarticular injections of PBSC plus hyaluronic acid (HA) were then carried out. HA is a naturally occurring substance found in the body's to improve cartilage health and repair, seed up the development of immature cells into chondrocytes, and reduce joint inflammation.

These studies highlighted the potential of their method for the successful regeneration of damaged their procedure effectively and simultaneously treat multiple lesions, it was also shown to treat 'kissing' boneon-bone lesions (not rare but serious njuries where there is damaged tissue on both sides of
the bone joint).

As Jee explains, 'the science, surgical technique, harvesting, and storage and the postoperative remabilitation programme that encompasses this innovative technology have been developed to enable high quality regeneration and repair'.

A stem cell is an immature, basic cell that has yet to specialise, or develop, meaning it has the potential to become any of the many types of cells in our bodies. For example, an undifferentiated stem cell may become a nerve, liver, heart, or blood cell, depending on the conditions they develop under. This regenerative potential. safely harvested, but the researchers' regeneration therapy coutd improve choice. Not only are they easily and their effective cartilage healing potential. Notably. PBSC can be cryopreserved for later use.
On the basis of
the team's earlier the team's earlier conducted to establish whether PBSC he functional outcomes for patients and reduce their knee pain. PBSC therapy was compared to patients

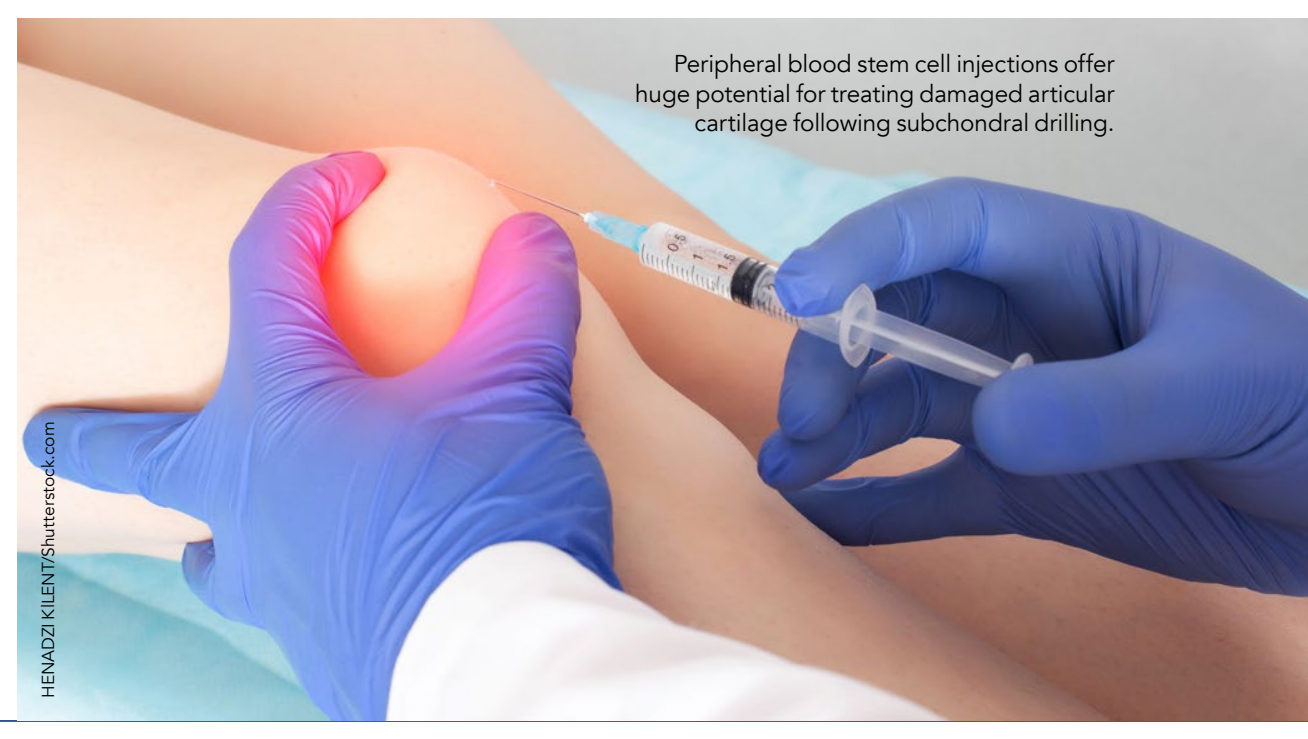




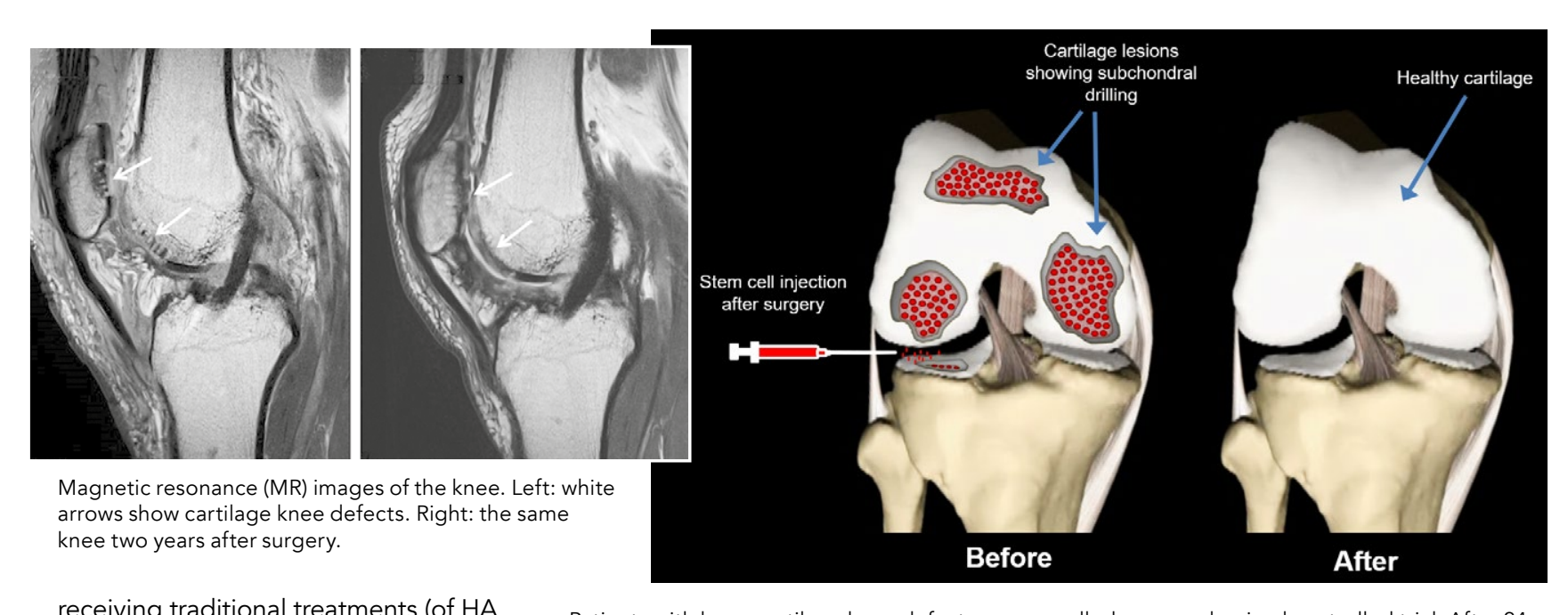

receiving traditional treatments (of $\mathrm{HA}$ injections and physiotherapy). Patien with large cartilage knee defects (classified as $\geq 3 \mathrm{~cm}^{2}$ ) were recruit to a 24-month dual-centre, open-
label phase IIB randomised controlled trial (RCT). A group of 69 patients trial (RCT). A group of 69 patients years, with half randomly receiving HA and PBSC injections following arthroscopic subchondral drilling and the other half receiving the $H A$ injections to act as a control group. Both patient groups also received post-treatment physiotherapy.

After 24 months, the patients' knee pain was scored, and their rate of cartilage repair was recorded. Primary

Patients with large cartilage knee defects were enrolled on a randomised controlled trial. After 24
months, patients' knee pain was scored, and the rate of cartiliage repair was recorded.

Notably, more than $70 \%$ of patients with 'unmet medical needs' who received the articular regeneration treatment showed significant improvement.

As a secondary outcome measure, the function, measured using standardised researchers used the 'MOCART' score IKDC scores (a knee-specific measure (Magnetic resonance Observation of of symptoms, function, and sports activity). Pain reduction was also measured, using KOOS scores (which assess the health, symptoms, and functionality of the knee).

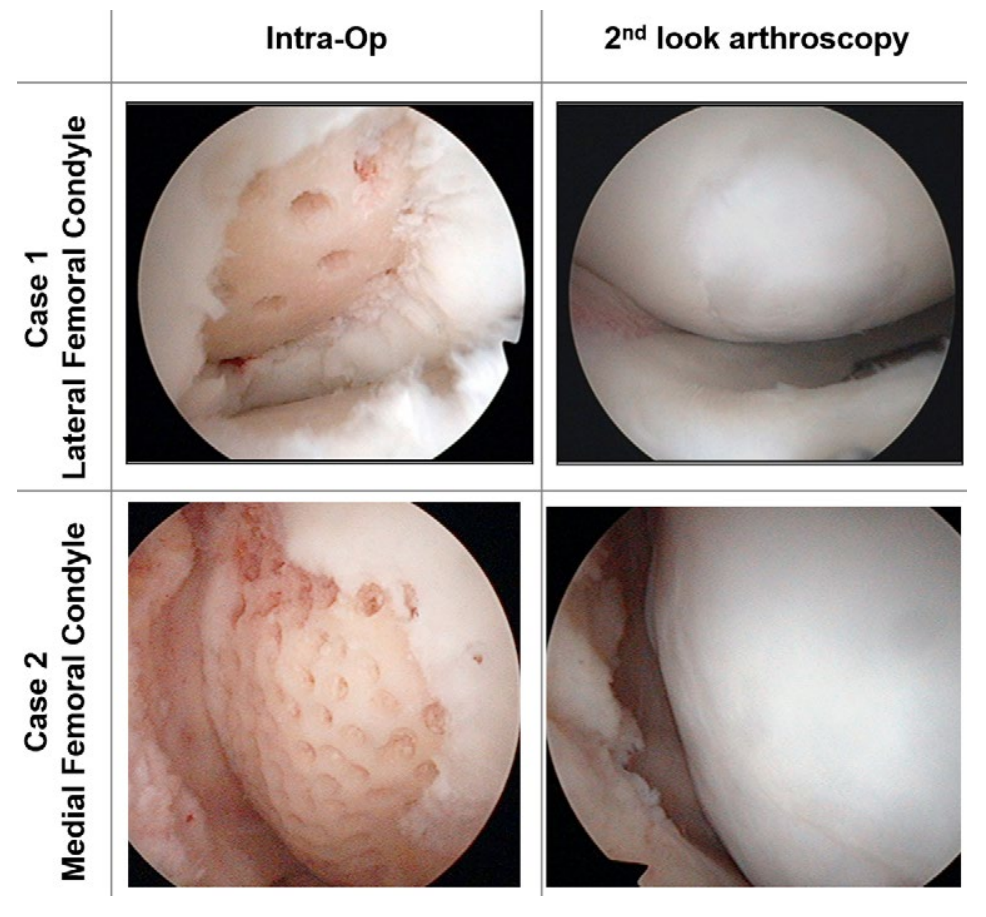

Left panels: Arthroscopici images of the knee following subchondral drilling.
Right panels: Arthroscopicimages at two years following surgery, showing fully regenerated cartilage.
Magnetic resonance Observation of Cartilage Repair Tissue) as a way to revealed there were no unexpected or adverse events throughout the duration of the study.

The interim analysis yielded following articular regeneration treatment, patients showed statistical to the HA control group. Only 12 months following treatment, the regeneration treatment group showed significant improvement across all measures: knee pain, knee function, and cartilage repair. Notably, more than $70 \%$ of patients who received the articular regeneration treatment showed significant improvement.

\section{THE POTENTIAL OF} REGENERATION THERAPY Articular regeneration treatment offers a safe and highly effective way of treating patients with massive cartilage defects. The researchers are now aiming Excitingly the resace 3 trial in 2022 . that their regesch team beleve could be applicable to other joints. promising results. After just six months

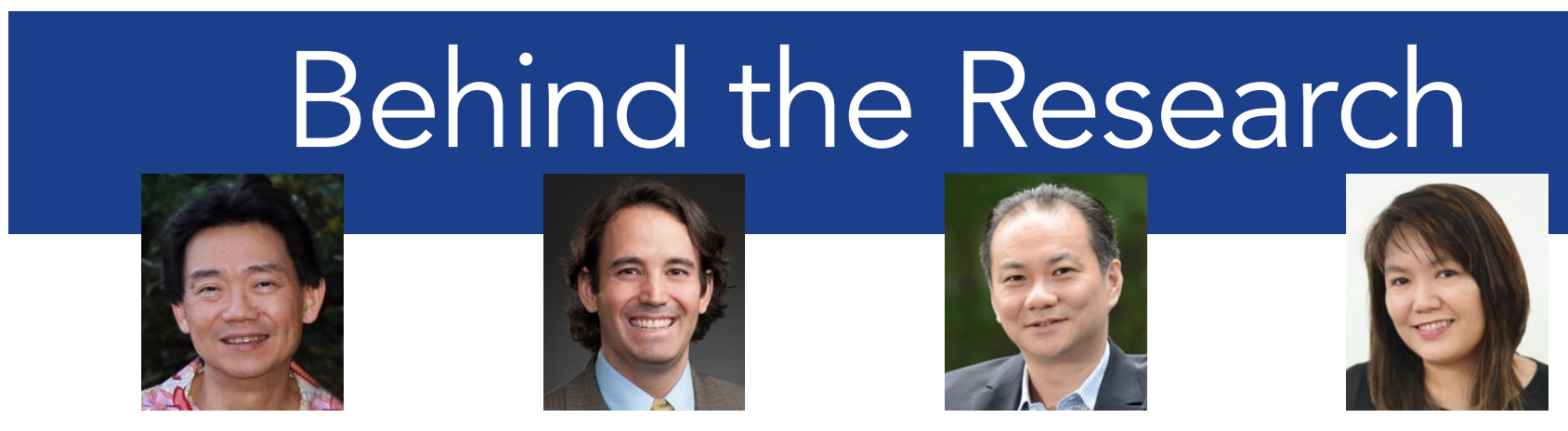

Dr Khay-Yong Saw

Dr Adam Anz

Dr Reza Ng

Dr Caroline Jee

Dr Saw, Dr Reza and Dr Jee E: enquiry@klsmc.com T: +60320961033 W: www.klsmc.com $\quad$ fo @klsmc Dr Anz:anz.adam.w@gmail.com T: +18509168476 @ @adamanzmd @ @adamanzmd A @adamanzmd

\section{Detail}

\section{Address}

Dr Saw, Dr Reza and Dr Jee

7th Floor, Wisma Perintis

47 , Jalan Dungun

Damansara Heights

50490 Kuala Lumpur

Malaysia

Dr Anz

Andrews Research and Education Foundation (AREF)

Sports Medicine Orthopaedic Surgery

GulfBrezecze Phy

Gulf Breeze
Florida 32561

USA

Bio

Dr Khay-Yong Saw is the founder and a consultant orthopaedic surgeon at KLSMC, Malaysia. He specialise chondrogenesis together with bone and soft tissse regeneration. He has received numerous awards, publications and patents for this pioneering technology and on his novel medical devices.

Dr Adam Anz is a consultant orthopaedic surgeon at the AREF in Pensacola, Florida, USA. His clinical and rese the focus has settled on knee, shoulder, and hip injuries as well as the development of innovative biologic treatments. He is one of the principal investigators on the cartilage regeneration study.

Dr Reza $\mathrm{Ng}$ received his education in medicine from Belgium with a specialist degree in orthopaedics. He is actively involved in stem cells research and its clinical application in treating orthopaedic degenerative diseases on the cartilage regeneration study.

Dr Caroline Jee manages all applied and fundamental She has successfully secured a number of governmentio. grants and awards. She holds ten international patents, is a chartered engineer (CEng), and chartered scientist (CSci).

Funding

- City of Gulf Breeze, Florida, and the State of Florida, USA - Agensi hnovasi Malaysia, Malaysia

\section{Research Objectives}

The development of stem-cell-based articular regeneration therapy for massive cartilage defects.

\section{References}

Saw, K-Y, et al, (2021) Arthroscopic Subchondral Drilling
Followed by lijection of Peripheral Blood Stem Cells and Hyaluronic Acid Showed Improved Outcome Compared to

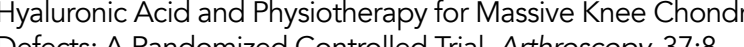
2502-2517. doi. org/10.1016/6/;arthro.2021.01.067

Saw, K-Y, etal, (2015) High Tibial Osteotomy in Combination With Chondrogenesis Atter Stem Cell Therapy: A Histologic Rerg/10.1016/:iarthro.2015.03.038

Sav, K-Y, et al, (2013) Articular Cartilage Regeneration With

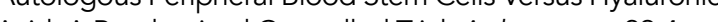
Acid: A Randomized Controlled Trial. Arthroscopy, 29.4 .
684-694. dx.doi.org/10.1016/:arthro.2012.12.008

Saw, K-Y, et al, (2011) Articular Cartilage Regeneration With Autologous Peripheral Blood Progenitor Cells and Hyaluronic Acid Atter Arthroscopic Subchondral Drilling: A Report
of 5 Cases With Histology. Arthroscopy, 27:4, 493-506. of 5 Cases With Histology. Arthros
doi:10.1016/jarthro.2010.11.054

\section{Personal Response}

How long do you think it will take for articular regeneration treatment to be the 'go-to' treatment for cartilage injury?

II This will take at least the next five years following subsequent regulatory approval. The process starts with the training of orthopaedic surgeons and familiarising the treating surgeons on the principles of chondrogenesis
with specialised surgical techniques and multiple postwith speciallsed surgical techiques and multiple postphysiotherapists on the importance of post-operative weightbearing exercises with movements of the operated joints is also an essential component. PBSC harvesting centres need to be established for cell preparations, chyopreservation, storage, and delivery. 